ARTICLE

Received 29 Apr 2016 | Accepted 16 Sep 2016 | Published 9 Nov 2016

DOl: $10.1038 /$ ncomms13278

OPEN

\title{
Unusually efficient photocurrent extraction in monolayer van der Waals heterostructure by tunnelling through discretized barriers
}

Woo Jong $\mathrm{Yu}^{1,2,3}$, Quoc An Vu 4,5,6, Hyemin Oh ${ }^{4,5}$, Hong Gi Nam ${ }^{4,5}$, Hailong Zhou', Soonyoung Cha ${ }^{7}$, Joo-Youn $\mathrm{Kim}^{7}$, Alexandra Carvalho ${ }^{8}$, Munseok Jeong ${ }^{4,5}$, Hyunyong Choi ${ }^{7}$, A.H. Castro Neto ${ }^{8}$, Young Hee Lee ${ }^{4,5,6}$

\& Xiangfeng Duan ${ }^{1,9}$

Two-dimensional layered transition-metal dichalcogenides have attracted considerable interest for their unique layer-number-dependent properties. In particular, vertical integration of these two-dimensional crystals to form van der Waals heterostructures can open up a new dimension for the design of functional electronic and optoelectronic devices. Here we report the layer-number-dependent photocurrent generation in graphene/MoS $/$ graphene heterostructures by creating a device with two distinct regions containing one-layer and seven-layer $\mathrm{MoS}_{2}$ to exclude other extrinsic factors. Photoresponse studies reveal that photoresponsivity in one-layer $\mathrm{MoS}_{2}$ is surprisingly higher than that in seven-layer $\mathrm{MoS}_{2}$ by seven times. Spectral-dependent studies further show that the internal quantum efficiency in one-layer $\mathrm{MoS}_{2}$ can reach a maximum of $65 \%$, far higher than the $7 \%$ in seven-layer $\mathrm{MoS}_{2}$. Our theoretical modelling shows that asymmetric potential barriers in the top and bottom interfaces of the graphene/one-layer $\mathrm{MoS}_{2}$ /graphene heterojunction enable asymmetric carrier tunnelling, to generate usually high photoresponsivity in one-layer $\mathrm{MoS}_{2}$ device.

\footnotetext{
${ }^{1}$ Department of Chemistry and Biochemistry, University of California, Los Angeles, California 90095, USA. ${ }^{2}$ Department of Electronic and Electrical Engineering, Sungkyunkwan University, Suwon 16419, Republic of Korea. ${ }^{3}$ Samsung-SKKU Graphene Center (SSGC), Suwon 16419, Republic of Korea. ${ }^{4}$ Center for Integrated Nanostructure Physics, Institute for Basic Science (IBS), Suwon 16419, Republic of Korea. ${ }^{5}$ Department of Energy Science, Sungkyunkwan University, Suwon 16419, Republic of Korea. ${ }^{6}$ Department of Physics, Sungkyunkwan University, Suwon 16419 , Republic of Korea. ${ }^{7}$ School of Electrical and Electronic Engineering, Yonsei University, Seoul 120-749, Korea. ${ }^{8}$ Centre for Advanced 2D Materials, National University of Singapore, 6 Science Drive 2, Singapore 117546, Singapore. ${ }^{9}$ California Nanosystems Institute, University of California, Los Angeles, California 90095, USA. Correspondence and requests for materials should be addressed to W.J.Y. (email: micco21@skku.edu) or to Y.H.L. (email: leeyoung@skku.edu) or to X.D. (email:xduan@chem.ucla.edu).
} 
$\mathrm{T}$ he two-dimensional (2D) layered materials such as graphene and semiconducting transition-metal dichalcogenides (TMDs) can exhibit peculiar electronic properties depending on their exact composition, thickness and geometry ${ }^{1-5}$. Furthermore, the broad possibility to combine different materials in van der Waals heterostructures (vdWHs) can create a new paradigm in materials science with unprecedented flexibility to integrate highly disparate materials and enable unique functions, as exemplified by the recent demonstration of vertical tunnelling transistors and vertical field-effect transistors for ultra-thin and flexible devices ${ }^{6-13}$ Graphene/multi-layer (ML)-TMD/graphene stack has also been shown to function as a unique photodiode for photocurrent generation or photodetection 7,12 . With a direct band gap in monolayer TMD and ultra-fast carrier transfer $(\sim 1 \mathrm{ps})$ rate $^{14}$, vdWHs with ultra-thin TMDs have attracted considerable interest for photovoltaic applications ${ }^{15,16}$ and ultra-fast photodetection ${ }^{17}$. However, classical charge transport theory for bulk semiconductors was often employed to interpret the electron transport in ultra-thin vdWHs even though it is well expected to exhibit completely different photocurrent generation characteristics 7,12 . Notably, an atomically thin graphene/WSe ${ }_{2} /$ $\mathrm{MoS}_{2}$ /graphene $p-n$ heterojonction has been investigated for photocurrent generation. However, Shockly-Read-Hall recombination and Langevin recombination in the $\mathrm{WSe}_{2} / \mathrm{MoS}_{2} p-n$ junction could compromise the carrier extraction performance ${ }^{13}$. The unique characteristics of ultrathin vdWHs are insufficiently explored to date. In particular, a systematic investigation of layernumber-dependent studies is lacking due to complications from highly variable nature of the van der Waals interfaces and extrinsic factors in creating vdWH devices.

Here we report a layer-number-dependent photocurrent generation in graphene/ $\mathrm{MoS}_{2} /$ graphene vdWHs by creating a device with two distinct regions containing one-layer (1L) and seven-layer (7L)- $\mathrm{MoS}_{2}$ to exclude extrinsic device factors. Significantly, we discover a surprisingly higher photoresponsivity and internal quantum efficiency (IQE) by going from ML MoS device to a monolayer device. The discretized electrostatic potential barriers were introduced to interpret the photocarrier tunneLling and extracting in ultrathin vdWHs.

\section{Results}

Photocurrent generation in graphene $/ 1 \mathrm{~L}-\mathrm{MoS}_{2} /$ graphene stack. Figure 1 shows the schematic illustration of graphene/1L-MoS $/$ graphene heterostructure device on $\mathrm{Si} / \mathrm{SiO}_{2}$ substrate. A focused laser was used to generate electron-hole pairs in the $\mathrm{MoS}_{2}$ layer, which can be separated by the asymmetric potential between the top graphene $\left(\mathrm{Gr}_{\mathrm{T}}\right) / \mathrm{MoS}_{2}$ and the bottom graphene $\left(\mathrm{Gr}_{\mathrm{B}}\right) / \mathrm{MoS}_{2}$ junction to produce photocurrent (Fig. 1a). Figure $1 \mathrm{~b}$ shows an optical image of a typical graphene/1L-MoS $/$ graphene vdWH device. The $8 \mu \mathrm{m}$ strip of $\mathrm{Gr}_{\mathrm{B}}$ is located below $1 \mathrm{~L}-\mathrm{MoS}_{2}$ flake (vertical strip). The $\mathrm{Gr}_{\mathrm{T}}$ layer is located directly on the $\mathrm{MoS}_{2}$ flake (horizontal strip) to overlap with $\mathrm{MoS}_{2}$ flake and the $\mathrm{Gr}_{\mathrm{B}}$.The photocurrent generation in our devices was mapped by scanning photocurrent microscopy, where a focused laser beam was rasterscanned over the sample, while the photocurrent was being measured (Fig. 1c). The spatially resolved photocurrent map reveals pronounced photocurrent generation in the overlapping region in the vertical stack. The current-voltage $\left(I_{\mathrm{ds}}-V_{\mathrm{ds}}\right)$ data obtained in the dark (blue line, Fig. 1d) and under $514 \mathrm{~nm}$ laser irradiation (red line, Fig. 1d) show a clear photoresponse in the $\mathrm{vdWH}$. In contrast to typical diode characteristics observed in graphene/ML- $\mathrm{MoS}_{2} /$ metal vdWHs due to asymmetric contact between top and bottom junction ${ }^{7,12}$, a linear transport curve is observed in the graphene/1L-MoS 2 /graphene heterostructure device, which can be attributed to direct tunnelling $\left(I_{\mathrm{DT}}\right)$ through ultrathin tunnelling barrier of $1 \mathrm{~L}-\mathrm{MoS}_{2}$

$$
I_{\mathrm{DT}}(V)=\frac{A_{\text {eff }} \sqrt{\mathrm{m} \varphi_{\mathrm{B}}} \mathrm{q}^{2} V_{\mathrm{ds}}}{\mathrm{h}^{2} d} \exp \left[\frac{-4 \pi \sqrt{m^{*} \varphi_{\mathrm{B}}} d}{\mathrm{~h}}\right]
$$

where $A_{\text {eff, }} \varphi_{\mathrm{B}}, q, m, m^{*}, d$ and $h$ are effective contact area, barrier height, electron charge, free electron mass, effective electron mass, barrier width ( $\mathrm{MoS}_{2}$ thickness) and Plank's constant, respectively ${ }^{18}$.

Based on the photocurrent response and input laser power, we can determine the photoresponsivity $\left(\mathrm{A} \mathrm{W}^{-1}\right)$ of the device. The photoresponsivity of $1 \mathrm{~L}-\mathrm{MoS}_{2}$ increases with decreasing the laser power could be attributed partly to absorption saturation in $\mathrm{MoS}_{2}$ and partly to the screening of a built-in electric field by the excited electrons in the conduction band of $\mathrm{MoS}_{2}$ (ref. 7). Importantly, the photoresponsivity of $1 \mathrm{~L}-\mathrm{MoS}_{2}$ increases with increasing the laser wavelength and a maximum photoresponsivity of $68 \mathrm{~mA} \mathrm{~W}^{-1}$ in $1 \mathrm{~L}-\mathrm{MoS}_{2}$ vdWHs was achieved at the wavelength of $633 \mathrm{~nm}$ and the laser power of $100 \mathrm{nW}$ (Fig. 1e), which exceeds that of previous studies on graphene/ML-MoS 2 /graphene vdWHs device $\left(\sim 30 \mathrm{~mA} \mathrm{~W}^{-1}\right.$ without plasmonic enhancement $)^{7,12}$. This performance difference is attributed to the number of layer difference between this work (monolayer $\mathrm{MoS}_{2}$ ) and previous reports ( $5 \sim 30 \mathrm{~nm} \mathrm{MoS}$ ), which will be further discussed by comparison of external quantum efficiency (EQE) and IQE between 1L-MoS 2 and $7 \mathrm{~L}-\mathrm{MoS}_{2}$ vdWHs in Fig. 2g,h.

Quantum efficiency between 1L-MoS 2 and $7 \mathrm{~L}-\mathrm{MoS}_{2}$. To further unambiguously illustrate the difference between monolayer and ML $\mathrm{MoS}_{2}$ device, we have created a vdWH device with two distinct regions with $1 \mathrm{~L}-$ and $7 \mathrm{~L}-\mathrm{MoS}_{2}$ in the same device (Fig. 2a). The $8 \mu \mathrm{m}$ strip of $\mathrm{Gr}_{\mathrm{B}}$ is located below the $\mathrm{MoS}_{2}$ flake (inside the dotted line) and the $\mathrm{Gr}_{\mathrm{T}}$ layer is located directly above the $\mathrm{MoS}_{2}$ flake (inside the solid line), to overlap with $\mathrm{MoS}_{2}$ flake and the $\mathrm{Gr}_{\mathrm{B}}$. The number of layers of $\mathrm{MoS}_{2}$ was then confirmed by Raman spectra measurements (Supplementary Fig. 1) and atomic force microscopic measurement. Figure $2 b$ display the photoluminescence (PL) mapping image of $1 \mathrm{~L}-\mathrm{MoS}_{2}$ and 7L-MoS 2 portion in Fig. 2a under an excitation wavelength of $532 \mathrm{~nm}(2.33 \mathrm{eV})$. The monolayer- $\mathrm{MoS}_{2}$ exhibited a much stronger PL compared with 7L-MoS , which is consistent with the direct band gap nature of $1 \mathrm{~L}-\mathrm{MoS}_{2}$ (band gap $\left.\sim 1.82 \mathrm{eV}\right)^{15,16}$ and the indirect band gap nature of $7 \mathrm{~L}-\mathrm{MoS}_{2}$ (band gap $\left.\sim 1.3 \mathrm{eV}\right)^{16}$. Two prominent PL peaks can be identified at $630 \mathrm{~nm}(1.96 \mathrm{eV})$ and $680 \mathrm{~nm}(1.82 \mathrm{eV})$ in the spectrum (Supplementary Fig. 2), corresponding to $\mathrm{A} 1$ and $\mathrm{B} 1$ direct excitonic transitions ${ }^{15}$.

The photocurrent map of the entire device clearly demonstrates that $1 \mathrm{~L}-\mathrm{MoS}_{2}$ region exhibited much more pronounced photocurrent than the 7L-MoS 2 region (Fig. 2c). The currentvoltage $\left(I_{\mathrm{ds}}-V_{\mathrm{ds}}\right)$ data obtained in the dark (black line, Fig. 3a) and under $514 \mathrm{~nm}$ laser irradiation (red line for $1 \mathrm{~L}-\mathrm{MoS}_{2}$ region and blue line for $7 \mathrm{~L}-\mathrm{MoS}_{2}$ region; Fig. 3a) show a clear photoresponse in the vdWH. The open-circuit voltage and a short-circuit current obtained in the $1 \mathrm{~L}-\mathrm{MoS}_{2}$ region are $110 \mathrm{mV}$ and $0.8 \mu \mathrm{A}$, both about one order of magnitude higher than those observed in the $7 \mathrm{~L}-\mathrm{MoS}_{2}(10 \mathrm{mV}$ and $0.08 \mu \mathrm{A}$, respectively). This is a rather surprising and counter-intuitive discovery, considering that the $7 \mathrm{~L}-\mathrm{MoS}_{2}$ should have significantly higher optical absorption than $1 \mathrm{~L}-\mathrm{MoS}_{2}$ (Fig. 3b and Supplementary Note 1).

The enhanced photoresponsivity of $1 \mathrm{~L}-\mathrm{MoS}_{2}$ may be attributed to two potential factors: higher photocarrier generation rate in the monolayer $\mathrm{MoS}_{2}$ and higher photocarrier extraction/collection efficiency. We will discuss the influence of each factor below, first by modelling the transition probability and subsequently by 
a

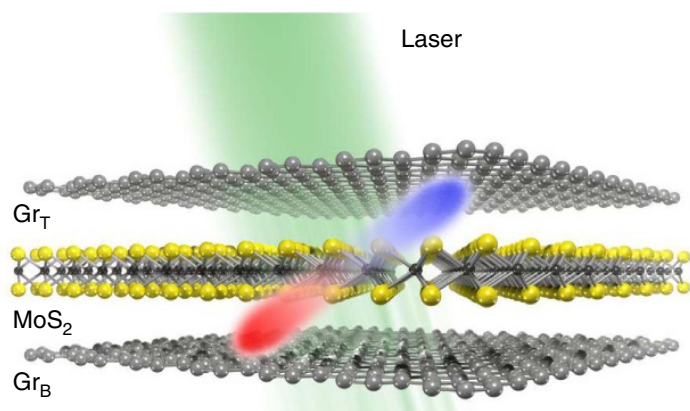

b

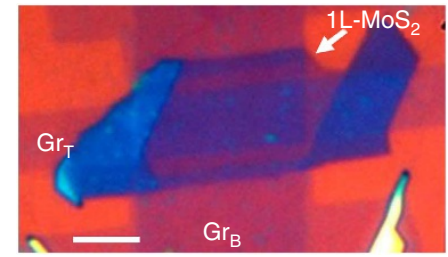

C

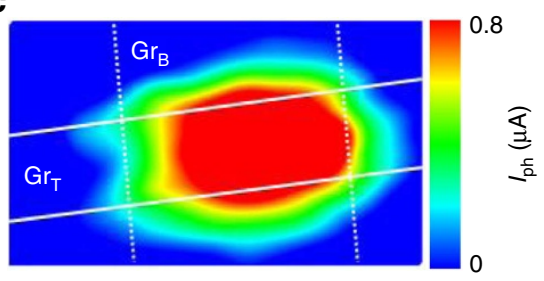

d

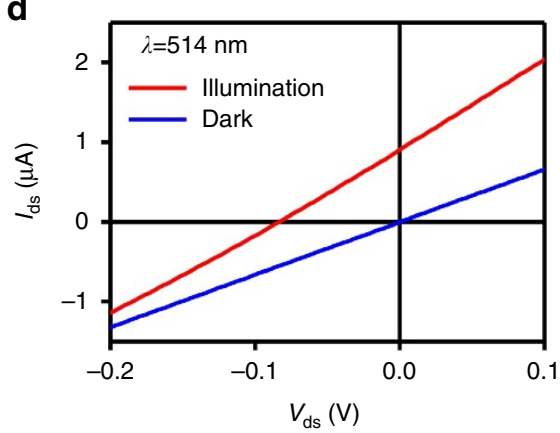

e

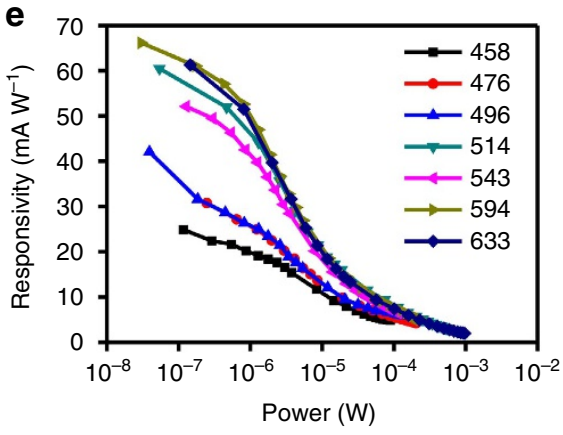

Figure 1 | Photocurrent generation in the vertical heterostructure of graphene/monolayer $\mathbf{M o S}_{\mathbf{2}} / \mathbf{g r a p h e n e}$ stack. (a) A schematic illustration of the side view of the device, with a monolayer $\mathrm{MoS}_{2}$ sandwiched between the top $\left(\mathrm{Gr}_{\mathrm{T}}\right.$ ) and bottom $\left(\mathrm{Gr}_{\mathrm{B}}\right)$ graphene electrodes. Hole (red particle) and electron (blue particle) are generated in the monolayer $\mathrm{MoS}_{2}$ layer by $514 \mathrm{~nm}$ laser and transferred to top and bottom graphene electrodes. (b) Optical image of the vertical heterostructures with monolayer $\mathrm{MoS}_{2}$ sandwiched between the top $\left(\mathrm{Gr}_{\mathrm{T}}\right)$ and bottom $\left(\mathrm{Gr}_{\mathrm{B}}\right)$ graphene electrodes. The scale bar is $4 \mu \mathrm{m}$. (c) Scanning photocurrent image taken under a $514 \mathrm{~nm}$ laser with an excitation power of $400 \mu \mathrm{W}$ and a spot size of $1 \mu \mathrm{m}$. The dotted and solid lines indicate the edge of the bottom and top graphene electrodes, respectively. (d) I-V characteristics of the device under laser illumination of $514 \mathrm{~nm}$ laser (red line) and dark (blue line). (e) Photoresponsivity of $1 \mathrm{~L}-\mathrm{MoS}_{2}$ heterostructures in various illuminated photon energies.

a

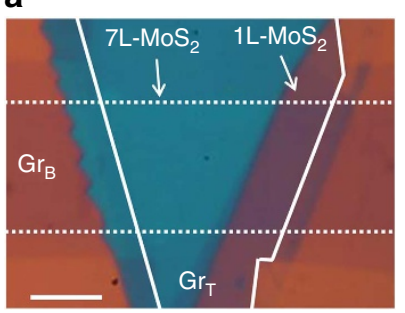

b

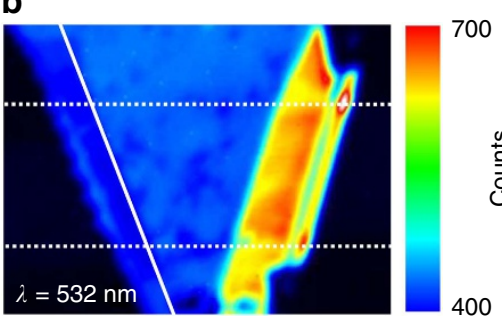

C

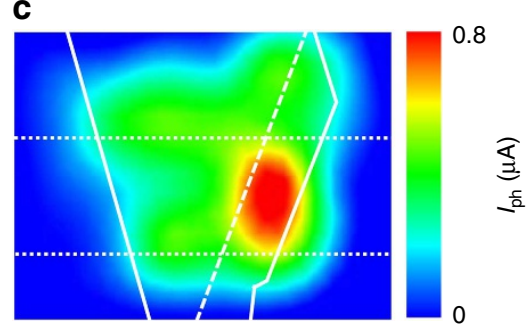

Figure 2 | Comparison of photocurrent generation in monolayer and $\mathbf{M L} \mathbf{M o S}_{\mathbf{2}}$ heterostructures. (a) Optical image of the $1 \mathrm{~L}$ MoS 2 vertical heterostructures with partially $7 \mathrm{~L} \mathrm{MoS} 2$ sandwiched between the $\mathrm{Gr}_{\mathrm{T}}$ and $\mathrm{Gr}_{\mathrm{B}}$ electrodes. The dotted line and solid lines indicate the edges of the $\mathrm{Gr}_{\mathrm{B}}$ and $\mathrm{Gr}_{\mathrm{T}}$ electrodes, respectively. Scale bar, $5 \mu \mathrm{m}$. (b) The PL image of the same device. (c) Scanning photocurrent image of the same device taken under a $514 \mathrm{~nm}$ laser with an excitation power of $400 \mu \mathrm{W}$ and a spot size of $1 \mu \mathrm{m}$. Dashed line indicates the boundary of $1 \mathrm{~L}-$ and $7 \mathrm{~L}-\mathrm{MoS}_{2}$.

comparing the internal and external quantum efficiencies. These two lines of reasoning will show that at peak IQE $(633 \mathrm{~nm}$ and $1.96 \mathrm{eV}$ ), the second factor (that is, the improved collection) dominates in our device.

In general, the spectral dependence of the photocarrier generation rate can be estimated from first principles, using the Fermi golden rule. The probability of transition from a valence band state $(v)$ to a conduction band state $(c)$ is thus given (in 2D) by

$$
W(\omega)=k_{2}(\omega) \frac{m_{\mathrm{e}}^{2} \omega^{2}}{2 \pi \mathrm{q}^{2} \hbar}
$$

where $k_{2}$ is the imaginary part of the dielectric function, $\hbar$ is the plank constant, $\omega$ is the frequency of the incident radiation, $m_{\mathrm{e}}$ and $q$ are the electron mass and charge. In $\mathrm{MoS}_{2}, k_{2}$ diverges because the conduction and valence bands run nearly parallel in some regions of the reciprocal space (that is, band nesting $\left.{ }^{19}\right)$. This can be noticed in Fig. 3c where we compare the imaginary part of the dielectric function, obtained from relativistic first-principles calculations, for $1 \mathrm{~L}$ and thick multilayer (bulk) $\mathrm{MoS}_{2}$. Thus, $W$ increases with the excitation energy up to the energy of the first peak, which is at $\sim 2.4 \mathrm{eV}$ for the bulk and $2.8 \mathrm{eV}$ for the monolayer, respectively ${ }^{19}$. The effect of band-nesting is more pronounced in monolayer, because this system is closer to the $2 \mathrm{D}$ limit and is 

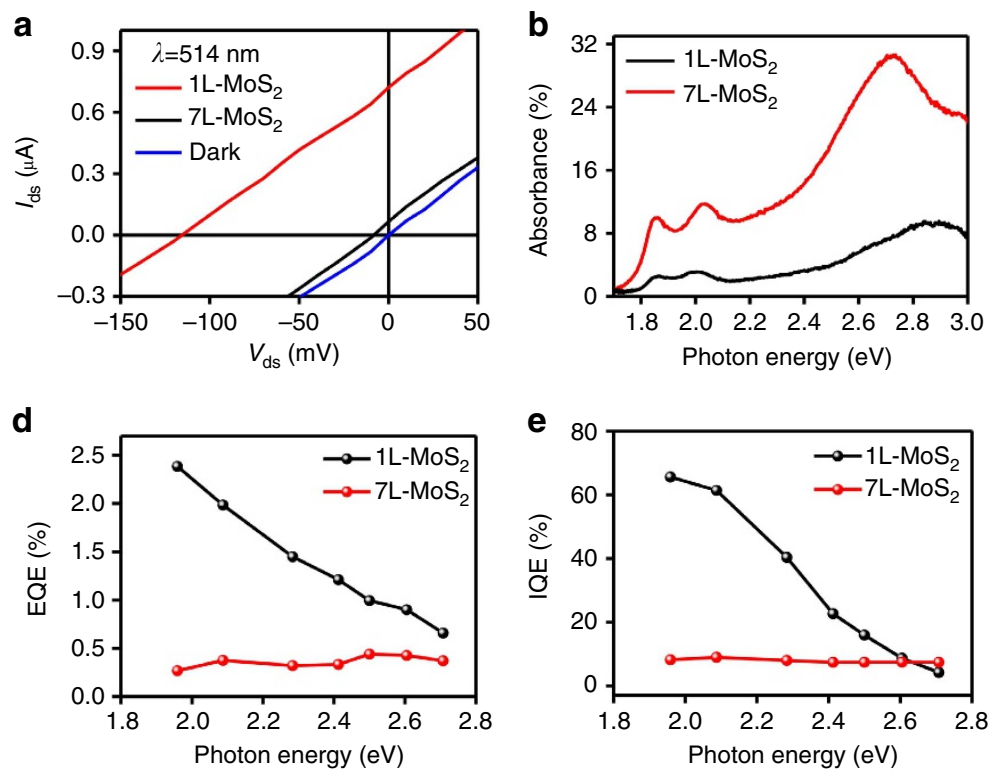
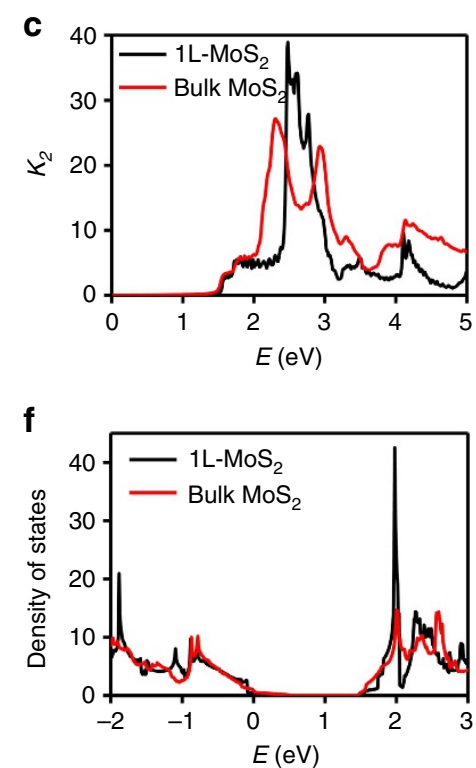

Figure 3 | Quantum effieciency between monolayer and $\mathbf{M L} \mathbf{M o S}_{\mathbf{2}}$ heterostructures. (a) $I_{\mathrm{ds}}-V_{\mathrm{ds}}$ characteristics of the device under laser illumination of $514 \mathrm{~nm}$ laser (red line for $1 \mathrm{~L}-\mathrm{MoS}_{2}$, blue line for $7 \mathrm{~L}-\mathrm{MoS}_{2}$ ) and dark (black line). (b) Absorption spectra of a $7 \mathrm{~L}-\mathrm{MoS}_{2}$ flake (red line) and $1 \mathrm{~L}-\mathrm{MoS} 2$ flake (black line). (c) Calculated imaginary part of the dielectric function $\left(k_{2}\right)$ of $1 \mathrm{~L}-$ and bulk $\mathrm{MoS}_{2}$ (as a model for thick ML). The effective thickness for the $1 \mathrm{~L}-\mathrm{MoS}_{2}$ was taken to be the inter-layer separation in $\mathrm{ML}^{-M o S_{2}}$. (d) EQE of $1 \mathrm{~L}-\mathrm{MoS}_{2}$ stack and $7 \mathrm{~L}-\mathrm{MoS}_{2}$ stack at a laser power of $100 \mu \mathrm{W}$. (e) Photon energy dependence of IQE at a laser power of $100 \mu \mathrm{W}$ (black line: $1 \mathrm{~L}-\mathrm{MoS}_{2}$; red line: 7L-MoS ). (f) Calculated density of states of $1 \mathrm{~L}-$ and ML-MoS 2 .

responsible for a raise in the absorption coefficient in that spectral region.

To separate the effects of enhanced photon absorption from carrier collection, we have also conducted the photocurrent studies under difference excitation photon energies and determined the corresponding $\mathrm{EQE}(\eta$, as defined by the number of carriers produced per incident photon ${ }^{20}$ ). The wavelengthdependent studies indicate the EQE of $1 \mathrm{~L}-\mathrm{MoS}_{2}$ increases with decreasing photon energy and can reach as high as $2.5 \%$ at $633 \mathrm{~nm}\left(1.96 \mathrm{eV}\right.$; Fig. 3d), whereas the EQE of $7 \mathrm{~L}-\mathrm{MoS}_{2}$ maintains a relatively low value of $0.3-0.4 \%$ throughout the entire measured wavelength range (Fig. $3 \mathrm{~d}$ ). Thus, the increased EQE does not follow the trend of $k_{2}$ that increases with increasing photon energy. This indicates that the enhanced absorbance is not the primary factor responsible for the maximum EQE. Other factors such as carrier collection should be considered.

Further insight is given by the IQE, which can be obtained by dividing the EQE by the optical absorbance of $7 \mathrm{~L}$ - and $1 \mathrm{~L}-\mathrm{MoS}_{2}$ (Fig. 3e). Importantly, the maximum IQE in $1 \mathrm{~L}-\mathrm{MoS}_{2}$ can reach up to $65 \%$, far exceeding to IQE in $7 \mathrm{~L}-\mathrm{MoS}_{2}(<7 \%)$. It is also noted that the IQE in $1 \mathrm{~L}-\mathrm{MoS}_{2}$ region steadily increases when the photon energy is reduced to approach the bandgap of $1 \mathrm{~L}-\mathrm{MoS}_{2}(1.82 \mathrm{eV}$ (ref. 15)). The highest IQE reached $\sim 65 \%$ at $633 \mathrm{~nm}(1.96 \mathrm{eV})$, very close to the optical absorption edge of $1 \mathrm{~L}-\mathrm{MoS}_{2}(1.82 \mathrm{eV})$. It is noteworthy that in this region $k_{2}$ is nearly independent on the thickness. According to the theoretical calculations, $1 \mathrm{~L}-\mathrm{MoS}_{2}$ has a much larger density of states near the band edges, at the valence band top due to the heavier hole masses, and near the conduction band edge, due to the presence of the $2 \mathrm{D}$ van-Hove singularities (Fig. 3f), increasing the probability of transfer to the graphene electrodes.

Photocarrier extraction mechanism. Photocarrier transport in bulk $\mathrm{MoS}_{2}$ is driven by diffusion and drift process, which is dictated by different band bending at $\mathrm{Gr}_{\mathrm{T}}$ and $\mathrm{Gr}_{\mathrm{B}} \mathrm{MoS}_{2}$ interface (originated from the difference of $\mathrm{Gr}_{\mathrm{T}}$ and $\mathrm{Gr}_{\mathrm{B}}$ doping densities) ${ }^{7,12}$ (Supplementary Fig. 3a). The photo-carrier extraction in atomically thin vdWHs is predicted to be completely different from that in bulk heterojunctions. Unlike bulk $\mathrm{MoS}_{2}$ having a continuous band bending (Supplementary Fig. 3a), atomically thin vdWH has discrete energy states in the vertical direction (Supplementary Fig. 3b). The classical semiconductor charge transport model-the charge drifts downhill following the band slope-cannot describe photocurrent generation phenomena via such discrete energy states in vdW heterojunctions. To this end, we introduce a tunnelling transport model to describe the photocarrier tunnelling through electrostatic potential barriers formed at the atomically thin vdW heterojunctions. Electrostatic potential barriers of $1 \mathrm{~L}-\mathrm{MoS}_{2}$ heterostructures are calculated by density functional theory (DFT; Supplementary Fig. 4 and Supplementary Note 2). Large electrostatic potential barriers are discretely constructed between $\mathrm{Gr}_{\mathrm{T}} / \mathrm{Gr}_{\mathrm{B}}$ and $\mathrm{MoS}_{2}$ interface. The barrier heights at the top and bottom junctions are symmetric because of symmetric environmental conditions imposed in the DFT calculations. In real device, the different environmental conditions of $\mathrm{Gr}_{\mathrm{T}}$ and $\mathrm{Gr}_{\mathrm{B}}$ result in asymmetric doping between $\mathrm{Gr}_{\mathrm{T}}$ and $\mathrm{Gr}_{\mathrm{B}}$. Doping characteristics of $\mathrm{Gr}_{\mathrm{T}}$ and $\mathrm{Gr}_{\mathrm{B}}$ were confirmed by $2 \mathrm{D}$ peak shift in Raman spectrum of another graphene/1L-MoS $/$ graphene heterostructure device (Supplementary Fig. 5 and Supplementary Note 3). With $n$ - or $p$-type doping, the 2D Raman band exhibits a red or blue shift, respectively. In our device, the $\mathrm{Gr}_{\mathrm{T}}$ is more $p$-type doped than $\mathrm{Gr}_{\mathrm{B}}$. Such doping difference generates internal field, leading to asymmetric barrier height between top and bottom junctions (Supplementary Fig. 6). In this way, the photoexcited electrons can effectively tunnel to lower barrier at $\mathrm{Gr}_{\mathrm{T}} / \mathrm{MoS}_{2}$ interface, whereas higher barrier at $\mathrm{Gr}_{\mathrm{B}} / \mathrm{MoS}_{2}$ interface can block the electron tunnelling. Asymmetric tunnelling to top and bottom barrier thus generates photocurrent in vdWH device. It is noteworthy that the delta-function potential barriers for hole carriers are formed at the graphene/ $\mathrm{MoS}_{2}$ interface, allowing hole tunnelling from $\mathrm{MoS}_{2}$ to $\mathrm{Gr}_{\mathrm{B}}$ by built-in electric field ${ }^{21}$. 


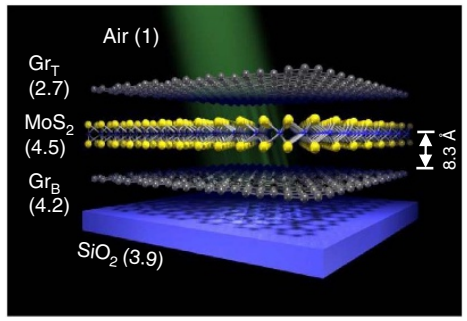

b

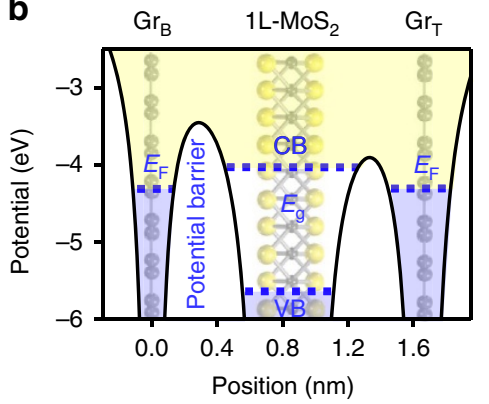

c

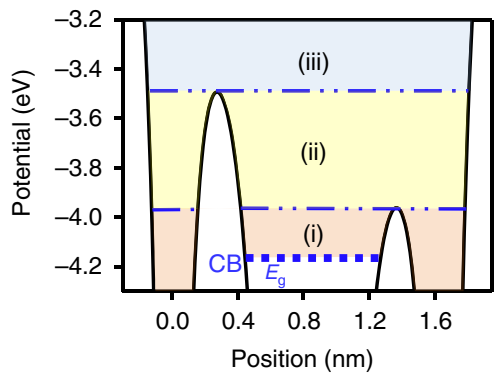

d

(i)

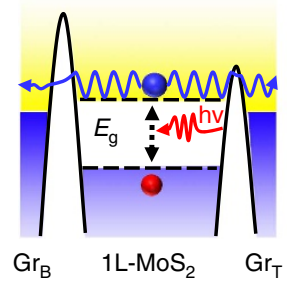

(ii)

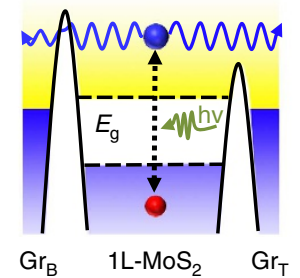

(iii)

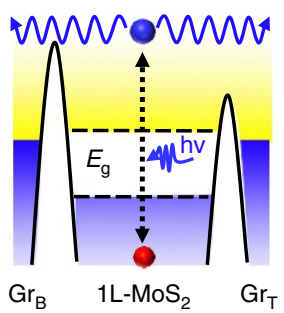

e

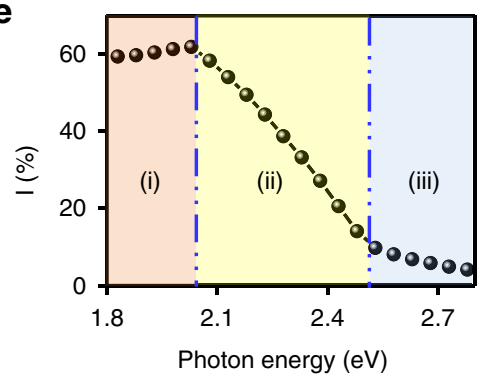

Figure 4 | Electrostatic potentials of monolayer $\mathbf{M o S}_{\mathbf{2}}$ heterostructure under different environments and photocarrier extraction mechanism.

(a) Schematic images of graphene/1L-MoS $/$ graphene heterostructures with $\mathrm{SiO}_{2}$ substrate and air environment. Dielectric constant of each layers is indicated in brackets. (b) Electrostatic potentials of graphene/1L-MoS 2 /graphene heterostructures including environmental condition (Supplementary Note 3). Green coloured areas are the potential energy barriers. $E_{\mathrm{f}}$ is the Fermi level of graphene. CB, VB and $E_{g}$ are conduction band, valence band and band gap of $\mathrm{MoS}_{2}$, respectively. (c) Electrostatic potentials near the conduction band of $1 \mathrm{~L}-\mathrm{MoS}_{2}$ heterostructures with three different potential barrier states. (d) Schematic images of photo-carrier tunnelling probabilities at the three different potential barrier states. (e) Calculated photocarrier extraction rate $\left(I=\left(T_{\mathrm{T}}-T_{\mathrm{B}}\right) /\left(T_{\mathrm{T}}+T_{\mathrm{B}}\right)\right)$

Furthermore, the different dielectric environment at the $\mathrm{Gr}_{\mathrm{T}}$ and $\mathrm{Gr}_{\mathrm{B}} / \mathrm{MoS}_{2}$ interface can significantly alter the electrostatic potential. Figure $4 \mathrm{a}$ shows the schematic illustration of the dielectric environment of the device. $\mathrm{The}_{\mathrm{B}} \mathrm{Gr}_{\mathrm{B}}$ is sandwiched between $\mathrm{SiO}_{2}$ substrate and $1 \mathrm{~L}-\mathrm{MoS}_{2}$, and the $\mathrm{Gr}_{\mathrm{T}}$ between $1 \mathrm{~L}-\mathrm{MoS}_{2}$ and air. The dielectric constant of $\mathrm{Gr}_{\mathrm{B}}$ (4.2) and $\mathrm{Gr}_{\mathrm{T}}$ (2.7) are determined by the standard approximation of $\varepsilon_{\mathrm{GrB}}=\left(\varepsilon_{1 \mathrm{~L}-\mathrm{MoS}_{2}}+\varepsilon_{\mathrm{SiO}_{2}}\right) / 2=4.2$ and $\quad \varepsilon_{\mathrm{GrT}}=\left(\varepsilon_{1 \mathrm{~L}-\mathrm{MoS}_{2}}+\varepsilon_{\mathrm{air}}\right) / 2=2.7$, respectively ${ }^{22,23}$, where $\varepsilon_{1 \mathrm{~L}-\mathrm{MoS}_{2}}=4.5$ (ref. 24).

The calculated electrostatic potentials with these dielectric constants are shown in Fig. 4b,c (calculation is shown in Supplementary Note 4$)^{25}$. Asymmetric electrostatic barriers are formed between the bottom $(\sim 3.5 \mathrm{eV})$ and top $(\sim 4.05 \mathrm{eV})$ junctions (Fig. $4 \mathrm{~b}, \mathrm{c}$ ). The photocarrier extraction rate $(I)$ can be determined by the difference of electron tunnelling probabilities between bottom and top junction barriers, which is normalized by the sum of tunnelling probability to top $\left(T_{\mathrm{T}}\right)$ and bottom junction barrier $\left.\left(T_{\mathrm{B}}\right)(I)=\left(T_{\mathrm{T}}-T_{\mathrm{B}}\right) /\left(T_{\mathrm{T}}+T_{\mathrm{B}}\right)\right)$. The potential barrier was sliced to thin square barriers with width of $0.01 \mathrm{~nm}$ and then the tunnelling probability was calculated through overall square barriers (Supplementary Fig. 7).

Based on this model, the photocarrier extraction rate $(I)$ strongly relies on the excitation photon energy (Fig. 4c,d). The tunnelling behaviour of excited electrons can be separated to three states, depending on the energy of excited electron, fully confined between $T_{\mathrm{T}}$ and $T_{\mathrm{B}}$, partially confined at the $T_{\mathrm{B}}$ and no confinement.

For fully confined between $T_{\mathrm{T}}$ and $T_{\mathrm{B}}$ (Fig. $4 \mathrm{~d}-\mathrm{i}$ ), the excited electrons in $\mathrm{MoS}_{2}$ can be transported to $\mathrm{Gr}_{\mathrm{T}}$ and $\mathrm{Gr}_{\mathrm{B}}$ by tunnelling through both sides of the potential barriers. The tunnelling probabilities through $T_{\mathrm{T}}$ or $T_{\mathrm{B}}$ is defined to $\exp \left(-2 \sqrt{\frac{2 m^{*(U-E)}}{\hbar^{2}}} a\right.$ ) (ref. 26), where $a$ is energy barrier width, $m^{*}$ is electron effective mass, $U$ is electrostatic barrier, $E$ is kinetic energy of electron and $h$ is the Planck's constant. The top junction allows for higher tunnelling probability due to smaller barrier height compared with the bottom junction. Consequently, electrons can transfer to the $\mathrm{Gr}_{\mathrm{T}}$ with positive $I$. The photocarrier extraction rate $(I)$ is not altered appreciably in response to the energy of excited electrons (or absorbed photon energy), because effective barrier height $(U-E)$ of the bottom and top junction changes by the similar magnitude (Fig. $4 \mathrm{e}-\mathrm{i}$ ).

For partially confined at the $T_{\mathrm{B}}$ (Fig. $4 \mathrm{~d}$-ii), the barrier at the bottom junction is higher than the kinetic energy of excited electrons. The tunnelling probability to $T_{\mathrm{B}}$ can be defined by $\exp \left(-2 \sqrt{\frac{2 m^{*(U-E)}}{\hbar^{2}}} a\right)$. On the other hand, the barrier at the top junction is lower than the energy of excited electron. The excited electrons transfer to $\mathrm{Gr}_{\mathrm{T}}$ over the barrier with a tunnelling probability of $\left(\frac{2 k_{\mathrm{Mos}_{2}}}{k_{\mathrm{CB}}+k_{\text {barrier }}}\right)^{2} \frac{k_{\text {barrier }}}{k_{\mathrm{Mos}_{2}}}$, where $k_{\mathrm{MoS}_{2}}=\sqrt{\frac{2 m^{*\left(E-U_{C B}\right)}}{\hbar^{2}}}$ $k_{\text {barrier }}=\sqrt{\frac{2 m^{*(E-U)}}{\hbar^{2}}}$, where $U_{\mathrm{CB}}$ is conduction band minimum of $\mathrm{MoS}_{2}$ (refs 21,26). The tunnelling probability through $T_{\mathrm{B}}$ increases with increase electron excitation energy $(E)$ because of the decrease of effective barrier height $(U-E)$, whereas the tunnelling probability to the $T_{\mathrm{T}}$ remains almost at 1 regardless of the electron energy. Consequently, the photocarrier extraction rate $(I)$ decreases in proportion to electron excitation energy (Fig. 4e-ii).

For no confiment case (Fig. $4 \mathrm{~d}$-iii), both $T_{\mathrm{B}}$ and $T_{\mathrm{T}}$ heights are lower than the energy of excited electrons; therefore, the tunnelling probability to $T_{\mathrm{T}}$ and $T_{\mathrm{B}}$ are defined by $\left(\frac{2 k_{\mathrm{MoS}_{2}}}{k_{\mathrm{CB}}+\mathrm{k}_{\mathrm{barrier}}}\right)^{2} \frac{k_{\text {barrier }}}{k_{\text {Mos }} \text {. The photocarrier extraction rate }}(I)$ decreases in proportion to the electron excitation energy (or absorbed photon energy; Fig. 4e-iii). 
$\mathrm{MoS}_{2}$ layer-dependent electrostatic potentials. The electrostatic potentials were calculated in terms of the number of $\mathrm{MoS}_{2}$ layers (Fig. 5). The region of fully confined between $T_{\mathrm{T}}$ and $T_{\mathrm{B}}$ case is expanded in response to the number of $\mathrm{MoS}_{2}$ layers due to the increased barrier heights at both sides. On the other hand, the region of partially confined at the $T_{\mathrm{B}}$ case is shrunken when the number of $\mathrm{MoS}_{2}$ layers increases due to the reduction of barrier height difference at both junctions. The region of no confinement case is also shrunken by increased barrier heights at both sides. Electrostatic potential is saturated over the $7 \mathrm{~L}-\mathrm{MoS}_{2}$ and behaves as bulk $\mathrm{MoS}_{2}$. The photocarrier extraction rates $(I)$ were calculated in terms of the electrostatic potentials.

Calculated photocarrier extraction rate. The carrier lifetime in direct gap $\left(1 \mathrm{~L}-\mathrm{MoS}_{2}\right)$ is shorter than that of indirect gap $\left(7 \mathrm{~L}-\mathrm{MoS}_{2}\right)$, which should be taken into account in recombination process during carrier drift. We have measured the carrier lifetime in direct gap $\left(1 \mathrm{~L}-\mathrm{MoS}_{2}\right)$ and indirect gap $\left(7 \mathrm{~L}-\mathrm{MoS}_{2}\right)$ based on transmission change in pump-probe measurement (Fig. 6a) and then calculated the normalized excited carriers $\left(\delta n_{x}\right)$ by initial excited carriers $\left(\delta_{0}\right)$ for $1 \mathrm{~L}-\mathrm{MoS}_{2}$ (direct gap, red line) and $\mathrm{ML}-\mathrm{MoS}_{2}$ (indirect gap, black line) along with carrier drift distance (nm; Fig. 6b and Supplementary Note 5). The normalized excited carrier density was then multiplied with photocarrier extraction rate (I) to clarify recombination difference in ML- $\mathrm{MoS}_{2}$. The $1 \mathrm{~L}-\mathrm{MoS}_{2}$ shows ultra-fast carrier transfer ( $\left.\sim 1 \mathrm{ps}\right)$ rate into graphene layer, much shorter than carrier lifetime in $1 \mathrm{~L}-\mathrm{MoS}_{2}$ (60 ps), resulting in highly efficient transfer of photoexcited carriers in $\mathrm{MoS}_{2}$ into graphene before recombination. Therefore, we multiplied photocarrier extraction rate (I) in $1 \mathrm{~L}-\mathrm{MoS}_{2}$ by $100 \%$ of normalized excited carrier density. Figure $6 \mathrm{c}$ clearly reveals that the photocarrier extraction rate $(I)$ reaches around $65 \%$ when the number of $\mathrm{MoS}_{2}$ layers is reduced from $7 \mathrm{~L}-\mathrm{MoS}_{2}$ to $1 \mathrm{~L}-\mathrm{MoS}_{2}$ and the photon energy approaches the bandgap $(1.82 \mathrm{eV})$. The calculated $I$ are in good agreements with measured IQE of $1 \mathrm{~L}-\mathrm{MoS}_{2}$ (black dashed line in Fig. 6d) and $7 \mathrm{~L}-\mathrm{MoS}_{2}$ heterostructures (red dashed line in Fig. 6d).

I- $\boldsymbol{V}$ characteristics. Previous studies have demonstrated an external gate voltage can be used to effectively modulate the a

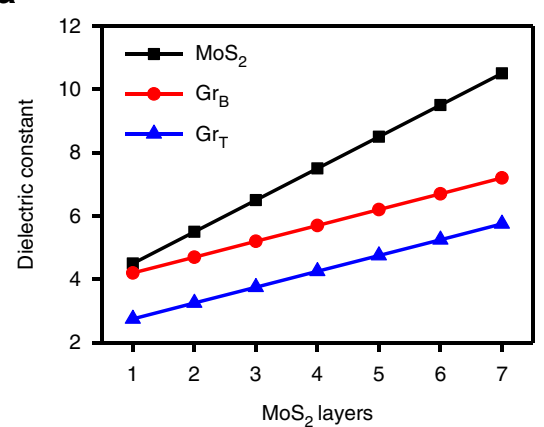

d

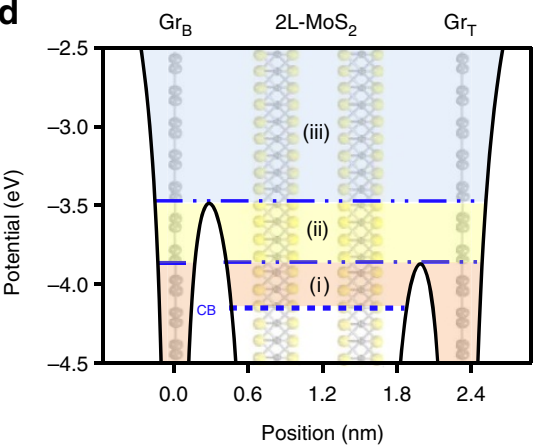

g

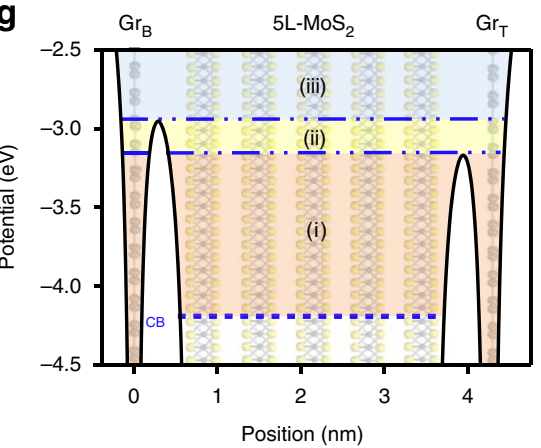

b

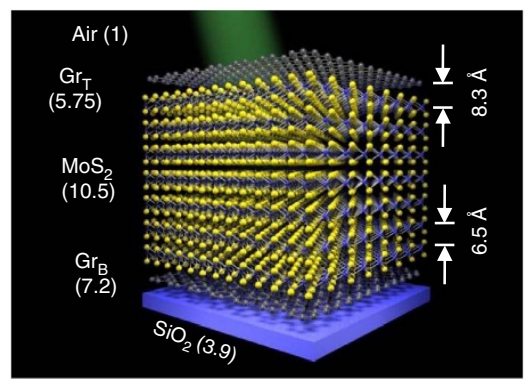

$\mathbf{e}$

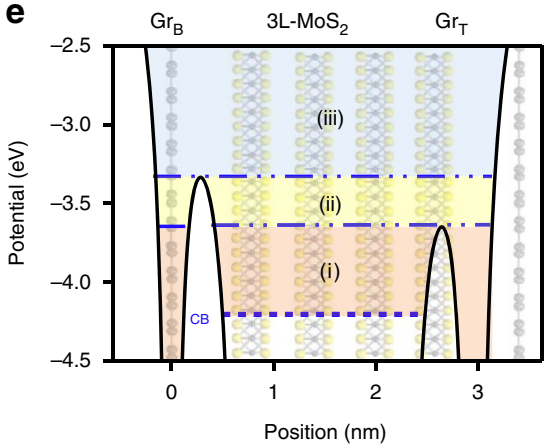

h

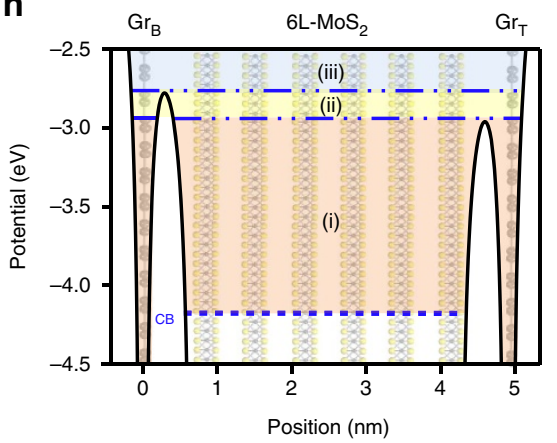

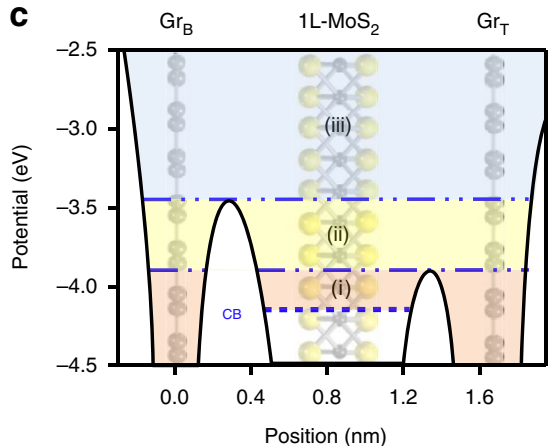

f
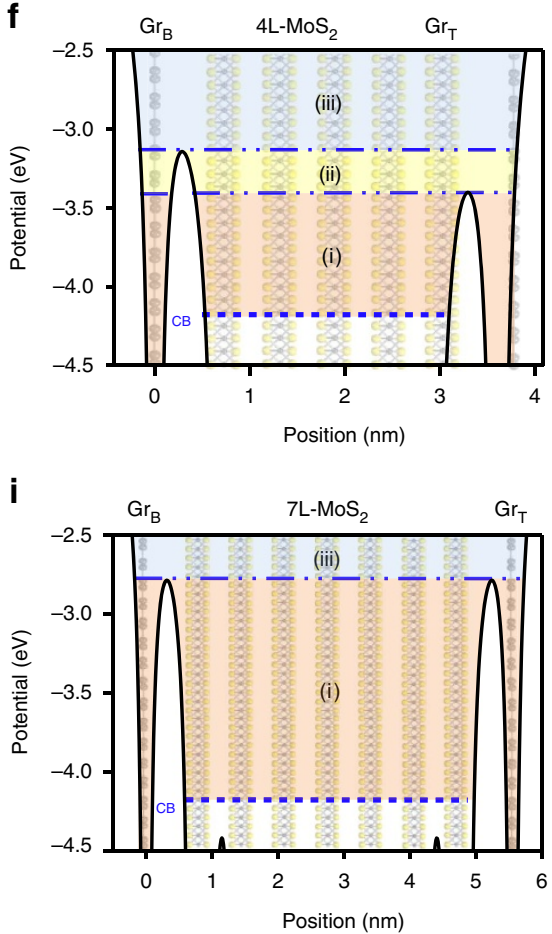

Figure 5 | $\mathbf{M o S}_{\mathbf{2}}$ layer-dependent electrostatic potentials of vdWHs. (a) $\mathrm{MoS}_{2}$ layer-dependent dielectric constant of $\mathrm{MoS}_{2}, \mathrm{Gr}_{\mathrm{T}}$ and $\mathrm{Gr}_{\mathrm{B}}$. (b-h) $\mathrm{MoS}_{2}$ layer-dependent electrostatic potentials of vdWHs. (i) Schematic images of graphene/7L-MoS $/$ graphene heterostructures with $\mathrm{SiO}_{2}$ substrate and $\mathrm{Air}$ environment. Dielectric constant of each layers are indicated in brackets. 
a
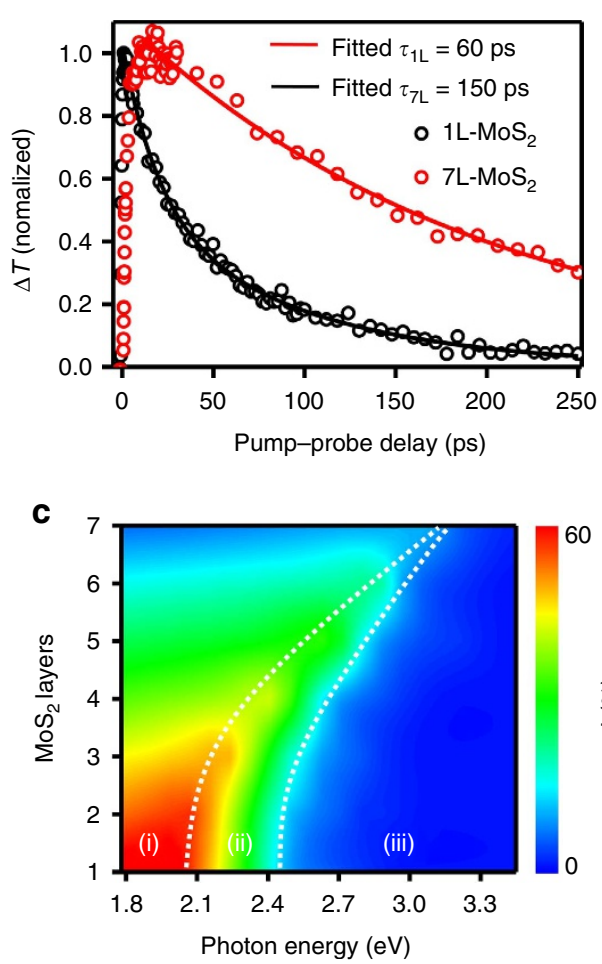

b

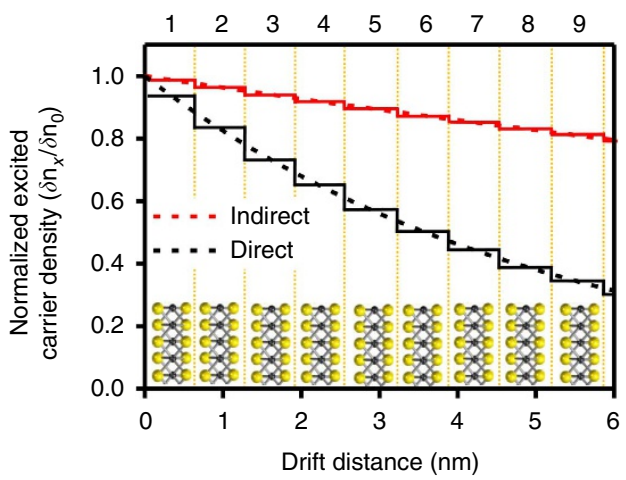

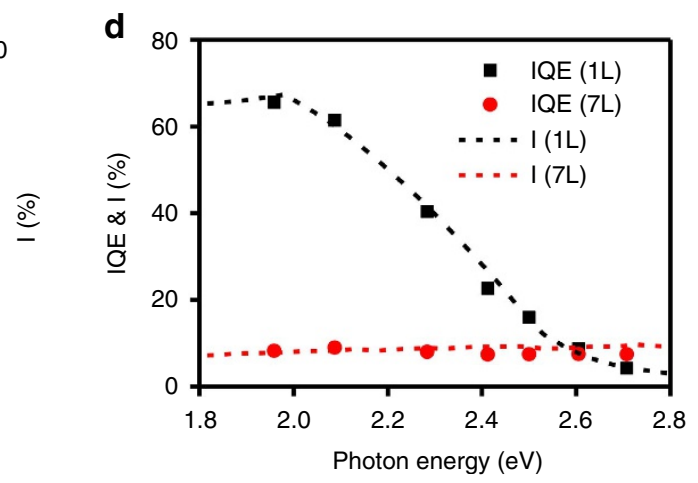

Figure 6 | Calculated photocarrier extraction rate of vDWHs with various number of $\mathbf{M o S}_{\mathbf{2}}$ layers. (a) Normalized dynamics of differential transmission $\left(\Delta T / T_{0}\right)$ of $1 \mathrm{~L}-\mathrm{MoS}_{2}$ and $7 \mathrm{~L}-\mathrm{MoS}_{2}$ for pump fluence (dots) and fitting lines. $\Delta T$ is the pump-induced probe transmission change and $T_{0}$ is the probe transmission without pump excitation for pump fluence. The decay lifetime of $1 \mathrm{~L}-\mathrm{MoS}_{2}\left(\tau_{\text {mono }}\right)$ and $7 \mathrm{~L}-\mathrm{MoS}_{2}\left(\tau_{\text {multi }}\right)$ obtained from the fitting.

(b) Normalized excited electron carriers $\left(\delta n_{x}\right)$ by initial excited electron carriers $\left(\delta n_{0}\right)$ for direct gap $\operatorname{MoS}_{2}$ (black line) and indirect gap MoS $($ red line) along with carrier drift distance $(\mathrm{nm})$. (c) $2 \mathrm{D}$ colour plot of photocarrier extraction rate as a function of the number of $\mathrm{MoS}_{2}$ layers and photon excitation energy. (d) Photon energy dependence of IQE (black dots: $1 \mathrm{~L}-\mathrm{MoS}_{2}$; red dots: $7 \mathrm{~L}-\mathrm{MoS}_{2}$ ) and calculated photocarrier extraction rate (black dashed line: 1L-MoS $;$ red dashed line: 7L-MoS 2 ).

Fermi energy of graphene and thus the potential offset and the driving force for carrier separation in graphene/ $\mathrm{MoS}_{2} /$ graphene devices ${ }^{7,12}$. We have also investigated gate voltage dependency of dark current (Supplementary Fig. 8) and photocurrent generation (Fig. 7) in the $1 \mathrm{~L}-\mathrm{MoS}_{2}$ and $\mathrm{ML} \mathrm{MoS}_{2}$ heterostructures. Overall, the $1 \mathrm{~L}-\mathrm{MoS}_{2}$ device showed considerably smaller modulation of short circuit current $\left(I_{\mathrm{sc}}\right)$ and open circuit voltage $\left(V_{\mathrm{oc}}\right)$ by an external gate voltage (Fig. 7a) than that of ML-MoS 2 (Fig. 7b). To understand the weak gate modulation in graphene/1L- $\mathrm{MoS}_{2} /$ graphene device, the band diagram of the $1 \mathrm{~L}-\mathrm{MoS}_{2}$ and ML- $\mathrm{MoS}_{2}$ devices at the positive and negative gate voltage are calculated (Supplementary Fig. 9 and Supplementary Note 6) and schematically illustrated in Fig. $7 c$, d. It is noted that the electrostatic screening of the $\mathrm{Gr}_{\mathrm{B}}$ layer is dependent on its carrier concentration, which can be varied by applied gate voltage. This factor is considered in the simulation and schematics. In general, the gate voltage can more efficiently modulate the $\mathrm{Gr}_{\mathrm{B}} E_{\mathrm{F}}$ than $\mathrm{Gr}_{\mathrm{T}} E_{\mathrm{F}}$ due to the partial screening of the gate field by the $\mathrm{Gr}_{\mathrm{B}}$ layer and sandwiched $\mathrm{MoS}_{2}$ layers. The larger gate modulation of the $T_{\mathrm{B}}$ than $T_{\mathrm{T}}$ can create additional potential offset or band slope that leads to change in $V_{\mathrm{oc}}$. In the ML-MoS ${ }_{2}$ device, the gate electric field primarily modulates the Fermi level $\left(E_{\mathrm{F}}\right)$ of the $\mathrm{Gr}_{\mathrm{B}}$ due to the relatively strong electrostatic screening by ML- $\mathrm{MoS}_{2}$ (Fig. 7d), leading to a large modulation of the band slope in ML-MoS ${ }_{2}$ and thus a substantial modulation of the $V_{\mathrm{oc}}$ and photocurrent. In contrast for the monolayer device, the gate electric field can effectively modulate both bottom and $\mathrm{Gr}_{\mathrm{T}} E_{\mathrm{F}}$ because of much weaker screening effect by the ultra-thin
$1 \mathrm{~L}-\mathrm{MoS}_{2}$ (Fig. 7c). As a result, both top and bottom potential barrier are modulated together in the graphene/1L-MoS $/$ graphene device, leading to much smaller dependence of potential offset and photocarrier extraction rate on the gate voltage.

\section{Discussion}

In summary, we have reported an unusually efficient photocarrier generation from monolayer $\mathrm{MoS}_{2}$ in graphene/ $\mathrm{MoS}_{2} /$ gaphene vdWHs. Owing to intrinsically slow layer-to-layer charge transport in TMD materials, the carrier mobility in the vertical direction is usually several orders of magnitudes lower than that in lateral direction, which can seriously slow down the charge separation process in the vertical heterostructures, leading to undesired photocarrier recombination in $\mathrm{MoS}_{2}$ before they arrive at the current collector. Importantly, we show that this drawback can be largely overcome by using atomically thin $\mathrm{MoS}_{2}$ to enable an unusually high photoresponsivity up to $68 \mathrm{~mA} \mathrm{~W}^{-1}$ with $65 \%$ of IQE in monolayer $\mathrm{MoS}_{2}$. This was congruent with carrier tunnelling through asymmetric electrostatic potential barriers that exist discretely in vdWHs with monolayer $\mathrm{MoS}_{2}$. Conversely, the symmetric potential barrier in ML $\mathrm{MoS}_{2}$ reduces photocurrent via carrier tunnelling. The proposed quantum mechanical-based tunnelling mechanism provides a new theoretical framework to understand van der Waals interaction in vdWHs and to design the next generation of atomically thin optoelectronics including photodetectors and photovoltaic devices. 
a

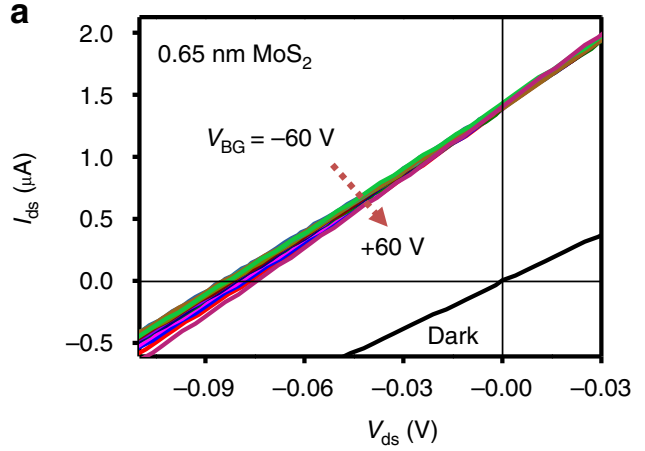

C

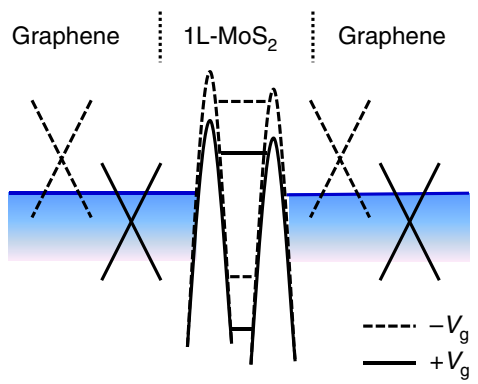

b

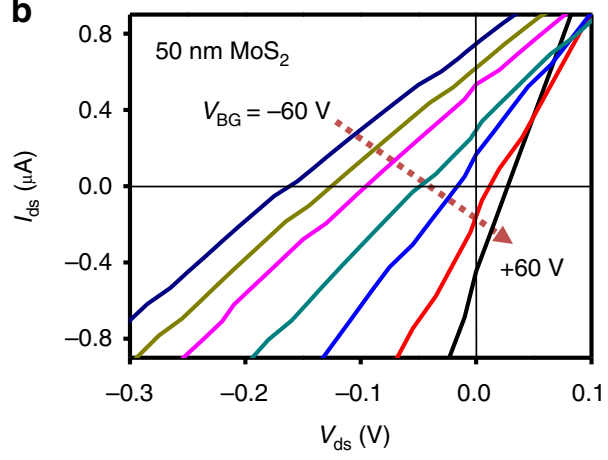

d Graphene $\mathrm{ML}-\mathrm{MoS}_{2} \quad \mathrm{Graphen}^{\mathrm{a}}$

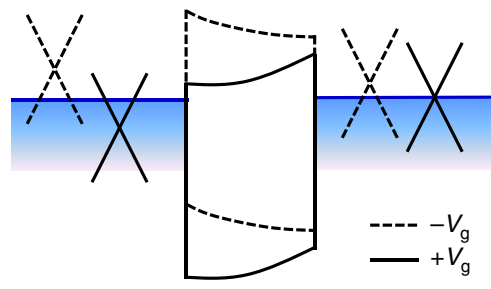

Figure 7 | I-V characteristics of monolayer and $\mathbf{M L} \mathbf{M o S}_{\mathbf{2}}$ (a,d) I-V characteristics of (a) graphene/1L-MoS $/$ graphene and (b) graphene/ML MoS 2 $(50 \mathrm{~nm}) /$ graphene under laser illumination of $514 \mathrm{~nm}$ laser and dark (black line, $V_{\mathrm{g}}=0 \mathrm{~V}$ ). Schematic band diagram for (c) graphene/1L-MoS $2 / g r a p h e n e$ and (d) graphene/ML $\mathrm{MoS}_{2}$ /graphene at the negative $V_{\mathrm{g}}$ (dashed line) and positive $V_{\mathrm{g}}$ (solid line).

It is noteworthy that the $2.5 \% \mathrm{EQE}$ achieved in our graphene/ $1 \mathrm{~L}-\mathrm{MoS}_{2}$ /graphene devices are comparable to the $2.4 \%$ value achieved in graphene/ $1 \mathrm{~L}-\mathrm{WSe}_{2} / 1 \mathrm{~L}-\mathrm{MoS}_{2} /$ graphene $p-n$ heterojonctions. Considering the absorbance in two layers stack $\left(\mathrm{MoS}_{2} / \mathrm{WSe}_{2}\right)$ is approximately twice of that in single layer $\left(\mathrm{MoS}_{2}\right)$, the IQE in our device is about twice higher. The lower IQE in in graphene $/ 1 \mathrm{~L}-\mathrm{WSe}_{2} / 1 \mathrm{~L}-\mathrm{MoS}_{2} /$ graphene $p-n$ heterojunctions may be partially attributed to Shockly-Read-Hall recombination and Langevin recombination, which is not present in graphene/1L- $\mathrm{MoS}_{2} /$ graphene devices.

\section{Methods}

The fabrication of the vertical heterostructures. For the fabrication of the vertical heterostructures of graphene/ $\mathrm{MoS}_{2} /$ graphene device, the graphene was grown with a chemical vapour deposition process ${ }^{27,28}$ and transferred onto $\mathrm{Si} / \mathrm{SiO}_{2}$ $(300 \mathrm{~nm} \mathrm{SiO})_{2}$ ) substrate, and patterned into $8 \times 30 \mu \mathrm{m}$ strips as the bottom electrode using a photolithography and oxygen plasma etching process. The $\mathrm{MoS}_{2}$ flakes were then transferred onto the graphene strips through a dry transfer approach ${ }^{27}$. The $\mathrm{Gr}_{\mathrm{T}}$ electrode was transferred and patterned on the $\mathrm{MoS}_{2}$ flake and $\mathrm{Gr}_{\mathrm{B}}$. Directly overlapping graphene area was patterned and etched away, to avoid short circuit between the $\mathrm{Gr}_{\mathrm{T}}$ and $\mathrm{Gr}_{\mathrm{B}}$. The metal electrode, for probe contact or wire bonding purposes, was patterned on the bottom and $\mathrm{Gr}_{\mathrm{T}}$ electrodes by e-beam lithography followed by e-beam deposition of Ti/Au $(50 / 50 \mathrm{~nm})$.

Microscopic and electrical characterization. Electrical transport measurements were conducted with a probe station and a computer-controlled analogue-to-digital converter at room temperature. The scanning photocurrent measurements were conducted with the same electrical measurement system under a SP2 MP Film confocal microscope coupled with Ar/ArKr laser (wavelength 458, 476, 488, 496 and $514 \mathrm{~nm}$ ) and HeNe laser (543, 596 and $633 \mathrm{~nm}$ ). All optical measurements were carried out under ambient conditions at room temperature by using an inverted microscope coupled to a grating spectrometer with a charge-coupled device camera. The optical beams were focused on the sample with a spot diameter of $\sim 1 \mu \mathrm{m} . \mathrm{MoS}_{2}$ heterostructures were excited with a cw solid-state laser at a wavelength of 405 and $532 \mathrm{~nm}$. A low laser power of $\sim 100 \mu \mathrm{W}$ was used to avoid heating and PL saturation. The PL of $\mathrm{MoS}_{2}$ samples were calibrated by using rhodamine $6 \mathrm{G}$ molecules as the standard. Raman spectroscopy (RM1000 microprobe; Renishaw) was used to characterize the $\mathrm{MoS}_{2}$ and graphene with a wavelength of $514 \mathrm{~nm}(2.41 \mathrm{eV})$ and a Rayleigh line rejection filter. As Raman scattering efficiency has usually little layer thickness dependence, the PL spectra normalized by Raman intensity reflects directly the luminescence efficiency ${ }^{16}$.
The DFT calculations. The DFT calculations were performed using the Quantum ESPRESSO code ${ }^{29}$. Geometry optimizations and total energy calculations are non-relativistic. Bandstructures calculated using a fully relativistic formalism are obtained using the atomic coordinates optimized in a similar non-relativistic calculation. The core electrons were described by norm-conserving, full relativistic pseudopotentials ${ }^{28}$ with nonlinear core-correction and spin-orbit information. The exchange correlation energy was described by the generalized gradient approximation, in the scheme proposed by Bueke et $a l^{30}$. The energy cutoff was 50 Ry.

The monolayer was modelled using a supercell consisting of $4 \times 4$ unit cells of the monolayer material, comprising a total number of 48 atoms. The lattice parameter $(a)$ used was optimized for the primitive unit cell. The supercell length along the direction perpendicular to the plane $c$ is taken to be twice the lattice parameter, except for preliminary bandstructure calculations performed using a smaller $c$, which are identified in the text. The Brillouin zone was sampled using a $2 \times 2 \times 1$ Monkhorst-Pack grid ${ }^{31}$.

The 7L material was modelled as a bulk crystal, using a $4 \times 4 \times 1$ supercell. The in-plane lattice parameter was taken from the monolayer calculation and the $c / a$ ratio was taken from experimental data ${ }^{32}$.

Ultrafast optical pump-probe spectroscopy. The femtosecond laser source is based on a $250 \mathrm{kHz}$ Ti:sapphire regenerative amplifier (Coherent RegA 9050), which provides a $1.55 \mathrm{eV}$ photon energy, $50 \mathrm{fs}$ pulse width and $6 \mu \mathrm{J}$ per laser pulse. The Reg A output pulse is separated into two pulses by a ratio of 7:3. The $70 \%$ laser pulse is used to generate $3.1 \mathrm{eV}$ pump-photon energy by second harmonic generation in a $1 \mathrm{~mm}$-thick beta barium borate crystal. The other $30 \%$ laser beam is focused in an ultra-clean sapphire disk to generate a white-light super-continuum, which serves as a probe pulse. Both the pump and the probe pulses are focused on the $1 \mathrm{~L}-\mathrm{MoS}_{2}$ and $7 \mathrm{~L}-\mathrm{MoS}_{2}$ samples by an objective lens (Mitutoyo M Plan Apo $\times 10)$ and the delay between the two pulses is controlled by a mechanical delay stage (Newport M-IMS300PP).

Data availability. The data that support the findings of this study are available from the corresponding author upon request.

\section{References}

1. Novoselov, K. S. et al. Two-dimensional atomic crystals. Proc. Natl Acad. Sci. USA 102, 10451-10453 (2005).

2. Novoselov, K. S. et al. Electric field effect in atomically thin carbon films. Science 306, 666-669 (2004).

3. Radisavljevic, B., Radenovic, A., Brivio, J., Giacometti, V. \& Kis, A. Single-layer $\mathrm{MoS}_{2}$ transistors. Nat. Nanotechnol. 6, 147-150 (2011). 
4. Wang, Q. H., Kalantar-Zadeh, K., Kis, A., Coleman, J. N. \& Strano, M. S. Electronics and optoelectronics of two-dimensional transition metal dichalcogenides. Nat. Nanotechnol. 7, 699-712 (2012).

5. Duan, X., Wang, C., Pan, A., Yu, R. \& Duan, X. Two-dimensional transition metal dichalcogenides as atomically thin semiconductors: opportunities and challenges. Chem. Soc. Rev. 44, 8859-8876 (2015).

6. Yu, W. J. et al. Vertically stacked multi-heterostructures of layered materials for logic transistors and complementary inverters. Nat. Mater. 12, 246-252 (2013).

7. Yu, W. J. et al. Highly efficient gate-tunable photocurrent generation in vertical heterostructures of layered materials. Nat. Nanotechnol. 8, 952-958 (2013).

8. Liu, Y. et al. Van der Waals heterostructures and devices. Nat. Rev. Mater. 1, 16042 (2016).

9. Ponomarenko, L. A. et al. Tunable metal-insulator transition in double-layer graphene heterostructures. Nat. Phys. 7, 958-961 (2011).

10. Britnell, L. et al. Field-effect tunneling transistor based on vertical graphene heterostructures. Science 335, 947-950 (2012).

11. Georgiou, T. et al. Vertical field-effect transistor based on graphene-WS heterostructures for flexible and transparent electronics. Nat. Nanotechnol. 8 100-103 (2013).

12. Britnell, L. et al. Strong light-matter interactions in heterostructures of atomically thin films. Science 340, 1311-1314 (2013).

13. Lee, C.-H. et al. Atomically thin p-n junctions with van der Waals heterointerfaces. Nat. Nanotechnol. 9, 575-681 (2014).

14. He, J. et al. Electron transfer and coupling in graphene-tungsten disulfide van der Waals heterostructures. Nat. Commun. 5, 5622 (2014).

15. Mak, K. F., Lee, C., Hone, J., Shan, J. \& Heinz, T. F. Atomically thin $\mathrm{MoS}^{2}$ : a new direct-gap semiconductor. Phys. Rev. Lett. 105, 136805 (2010).

16. Splendiani, A. et al. Emerging photoluminescence in monolayer $\mathrm{MoS}_{2}$. Nano Lett. 10, 1271-1275 (2010).

17. Massicotte, M. et al. Picosecond photoresponse in van der Waals heterostructures. Nat. Nanotechnol. 11, 42-46 (2016).

18. Lee, G. H. et al. Electron tunneling through atomically flat and ultrathin hexagonal boron nitride. Appl. Phys. Lett. 99, 243114 (2011).

19. Kozawa, D. et al. Photocarrier relaxation pathway in two-dimensional semiconducting transition metal dichalcogenides. Nat. Commun. 5, 4543 (2014).

20. Sze, S. M. \& Ng, K. K. Physics of Semiconductor Devices (Wiley, 2007).

21. Liboff, R. L. Introductory Quantum Mechanics (Addison Wesley, 2003).

22. Hwang, C. et al. Fermi velocity engineering in graphene by substrate modification. Sci. Rep. 5, 590 (2012).

23. Elias, D. C. et al. Dirac cones reshaped by interaction effects in suspended graphene. Nat. Phys. 7, 701 (2011).

24. Chen, X. et al. Probing the electron states and metal-insulator transition mechanisms in molybdenum disulphide vertical heterostructures. Nat. Commun. 6, 6088 (2015).

25. Livingston, J. D. Electronic Properties of Engineering Materials (Wiley \& Sons, Inc., 1999).

26. Neamen, D. A. Semiconductor Physics and Devices (McGraw-Hill, 2012).

27. Li, X. et al. Large-area synthesis of high quality and uniform graphene films on copper foils. Science 324, 1312-1314 (2009)

28. Zhou, H. et al. Chemical vapour deposition growth of large single crystals of monolayer and bilayer graphene. Nat. Commun 4, 2096 (2013).

29. Giannozzi, P. et al. The pseudopotentials used were either obtained from the Quantum ESPRESSO distribution or produced using the atomic code by A. Dal
Corso, that comes in the Quantum ESPRESSO distribution. J. Phys. -Cond. Matter 21, 395502 (2009).

30. Perdew, J. P., Burke, K. \& Ernzerhof, M. Generalized gradient approximation made simple. Phys. Rev. Lett. 77, 3865 (1996).

31. Monkhorst, H. J. \& Pack, J. D. Special points for Brillouin-zone integrations. Phys. Rev. B 13, 5188 (1976).

32. Wilson, J. A. \& Yoffe, A. D. The transition metal dichalcogenides discussion and interpretation of the observed optical, electrical and structural properties Adv. Phys. 18, 193-335 (1969).

\section{Acknowledgements}

X.D. acknowledges the support by ONR Award N00014-15-1- 2368. Y.H.L. acknowledges support by the Institute for Basic Science (IBS). W.J.Y. acknowledges HRD programme (number 20144030200580) of the KETEP grant funded by the KGMT and IENRF of Korea grant funded by the KGME, ST (NRF-2011-351-c00034). S.C. and H.C were supported by the National Research Foundation of Korea (NRF) grant funded by the Korea government (MSIP) (NRF-2015R1A2A1A10052520). A.H.C.N acknowledges the CRP award, "Novel 2D materials with tailored properties: beyond graphene" (NRF-CRP6-2010-05) and the Prime Minister's Office, Singapore, under its MediumSized Centre Programme.

\section{Author contributions}

X.D. conceived the research and designed the experiment. W.J.Y. performed most of the experiments including device fabrication, characterization, data analysis and theoretical analysis. Q.A.V. and Y.H.L. stacked graphene-monolayer $\mathrm{MoS}_{2}$-graphene by dry transfer process and performed Raman spectrum. H.O. and M.J. performed PL measurement. H.Z. synthesized the graphene. S.C., J.-Y.K. and H.C. performed optical pump-probe spectroscopy. A.C. and A.H.C-N. performed the DFT calculations. A.Y. performed absorption measurement. X.D., Y.H.L. and W.J.Y. wrote the paper. All authors discussed the results and commented on the manuscript.

\section{Additional information}

Supplementary Information accompanies this paper at http://www.nature.com/ naturecommunications

Competing financial interests: The authors declare no competing financial interests.

Reprints and permission information is available online at http://npg.nature.com/ reprintsandpermissions/

How to cite this article: $\mathrm{Yu}, \mathrm{W}$. J. et al. Unusually efficient photocurrent extraction in monolayer van der Waals heterostructure by tunnelling through discretized barriers. Nat. Commun. 7, 13278 doi: 10.1038/ncomms13278 (2016).

Publisher's note: Springer Nature remains neutral with regard to jurisdictional claims in published maps and institutional affiliations.

(c) (i) This work is licensed under a Creative Commons Attribution 4.0 International License. The images or other third party material in this article are included in the article's Creative Commons license, unless indicated otherwise in the credit line; if the material is not included under the Creative Commons license, users will need to obtain permission from the license holder to reproduce the material. To view a copy of this license, visit http://creativecommons.org/licenses/by/4.0/

(C) The Author(s) 2016 